\title{
Evaluation de la protection vaccinale contre la peste bovine au Cameroun. III. Evaluation globale
}

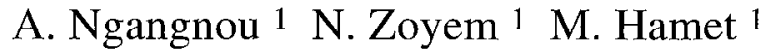 \\ S. Abdoulkadiri 1
}

Mots-clés

Bovin - Peste bovine - Peste des petits ruminants - Enquête - Vaccin - Test ELISA - Cameroun.

\begin{abstract}
Résumé
Le Laboratoire national vétérinaire (LANAVET) de Garoua (Cameroun) exécute le volet séro-surveillance dans le cadre de la Campagne panafricaine de lutte contre la peste bovine (PARC) depuis 1989. En 1993, I'enquête a porté sur 8517 sérums prélevés dans 286 troupeaux bovins âgés de 0 à 3 ans. Les troupeaux sondés ont été choisis par randomisation dans les six provinces à grand élevage bovin (Extrême-Nord, Nord, Adamaoua, Ouest, Nord-Ouest et Est). Les sérums ont été testés par la technique ELISA de compétition adaptée à la peste bovine par le projet conjoint FAO/AIEA. La prévalence observée a été de 54 p. 100. Deux mille dix sérums issus de 68 troupeaux à faible taux d'anticorps bovipestiques ont été testés par la technique ELISA de compétition, adaptée à la peste des petits ruminants (PPR). Seuls, 91 des sérums (4,5 p. 100) ont présenté des anticorps anti-virus de la PPR, cc qui n'augmente pas dc manière significative le niveau de protection du cheptel vis-à-vis de la peste bovine. D'autre part, la faiblesse de l'immunité post-vaccinale contre la peste bovine n'est vraisemblablement pas due à l'infection des bovins par le virus de la PPR. Les différences en anticorps entre les provinces visitées sont statistiquement significatives. Des recommandations sont émises en vue d'améliorer l'efficacité des campagnes de vaccination contre la peste bovine.
\end{abstract}

\section{INTRODUCTION}

Le Cameroun pratique depuis plusieurs années, des campagnes annuelles de vaccination contre la peste bovine. En vue d'en connaître les résultats, deux campagnes d'évaluation d'immunité post-vaccinale ont été menées dans deux provinces : Adamaoua en 1991, 62 p. 100 de prévalence en anticorps anti-peste bovine ; Nord en 1992, 66 p. 100 de prévalence en anticorps anti-virus de la peste bovine $(6,7)$. Les résultats sont done mitigćs.

Cependant, le taux de couverture des bovins vis-à-vis de la peste bovine pourrait être sous-estimé. En effet, les animaux porteurs d'anticorps anti-peste des petits ruminants (PPR) sont protégés contre la maladie. Or, les anticorps anti-PPR ne sont pas détectés par le test ELISA de compétition utilisé pour la mise en évidence des anticorps anti-peste bovine. D'autre part, l'infection des bovins par le virus de la PPR a parfois été évoquée par certains responsables des campagnes de vaccination contre la peste bovine pour expliquer le faible pourcentage de séroconversion suite à cette vaccination.

Deux êtudes ont donc été menées en 1993 dans les six provinces à grand effectif bovin :

- l'évaluation du pourcentage d'animaux porteurs d'anticorps bovipestiques dans la population bovine ;

1. Laboratoire national vétérinaire (LANAVET), B.P. 503, Garoua, Cameroun.
- l'évaluation du pourcentage d'animaux porteurs d'anticorps antiPPR dans la population bovine à faible taux d'anticorps bovipestiques.

Ces six provinces couvrent une superficie de $305290 \mathrm{~km}^{2}$, soit 64 p. $100 \mathrm{du}$ territoire national $\left(475000 \mathrm{~km}^{2}\right)$. Elles comportent 3787000 têtes de bovins, sur un total de 4360000 têtes, soit 87 p. 100, réparties en zébus Peuhls et arabes dans les provinces de l'Adamaoua, de l'Ouest, de l'Extrême-Nord, du Nord-Ouest, et en zébus Foulbé, Mbororo, Namchi dans les provinces du Nord, de l'Extrême-Nord (5).

\section{MATERIEL ET METHODES}

\section{Evaluation des anticorps hovipestiques dans la population bovine}

L'enquête a porté sur 8517 sérums prélevés dans 286 troupeaux. L'unité d'échantillonnage est le troupeau. Le nombre de troupeaux $\mathrm{n}$ à sonder a été déterminé suivant la formule classique d'échantillonnage aléatoire :

$\mathrm{n}=\mathrm{p}(1-\mathrm{q}) \mathrm{ET}^{2}(12)$ où :

- ET est l'erreur type pour un intervalle de confiance fixé à 95 p.100 et une précision de 10 p. $100(0,05)$

- p est la prévalence de base (50 p. 100$)$. 
Quatre degrés d'échantillonnage ont été définis :

ler degré : les provinces - six provinces ont été retenues : Adamaoua, Extrême-Nord, Nord, Est, Ouest, Nord-Ouest ;

2ème degré : tous les secteurs ont été retenus ;

3ème degré : tous les sous-secteurs à effectif important ont été retenus ;

4ème degré : les centres zootechniques et vétérinaires ( $C L V)$ ont été classés par ordre croissant d'effectif bovin.

Dans chaque province, chaque secteur, chaque sous-secteur et chaque centre, le nombre de troupeaux à visiter a été déterminé proportionnellement à la charge en bovins. Au niveau des CZV, les troupeaux ont été sélectionnés à l'aide de la table des nombres aléatoires. Dans chaque troupeau, 30 échantillons de sérums ont été prélevés (à raison de 10 échantillons par tranche d'âge : 0-1 an, 1-2 ans, 2-3 ans (11). Les sérums ont été identifiés par un code spécial, transportés au laboratoire dans des coffres isothermes avec glace et conservés à $-20^{\circ} \mathrm{C}$ en attendant d'être testés.

Les sérums ont été testés par la technique ELISA de compétition adaptée à la peste bovine par le projet conjoint $\operatorname{FAO/AIEA~}(1,4)$.

\section{Evaluation des anticorps anti-virus de la peste des petits ruminants dans la population bovine à faible taux d'anticorps bovipestiques}

L'enquête a porté sur 2010 sérums issus de 68 troupeaux ayant présenté une faible prévalence en anticorps bovipestitiques.

Les sérums ont été testés par la méthode ELISA de compétition PPR (kit fourni par le CIRAD-EMVT/CAMDA-OIE) (3).

\section{RESULTATS}

\section{Immunité post-vaccinale contre la peste bovine}

La figure 1 montre la répartition du cheptel dans les provinces visitées.

\section{Dans les provinces}

Le tableau I montre la prévalence en anticorps anti-peste bovine dans les différentes classes d'âge dans les six provinces visitées ; le tableau II montre la prévalence globale par province.

La province du Nord présente les meilleurs résultats bruts (64 \pm 5 p. 100 séropositifs vis-à-vis de la pestc bovinc), suivic de la province de l'Adamaoua ( $63 \pm 2$ p. 100 de séropositifs). Les résultats les moins bons sont enregistrés dans les provinces de l'Extrême-Nord et du Nord-Ouest $(44 \pm 2$ et $30 \pm 3$ p. 100 d'animaux positifs respectivement).

Les différences de prévalence en anticorps anti-peste bovine entre les six provinces sont statistiquement significatives (chi-2 $=203$; d.d.1. $=5 ; \mathrm{p}<0,05)$.

\section{Dans les tranches d'âge}

Le tableau I montre que le taux d'immunité post-vaccinale est inférieure à 50 p. 100 chez les sujets de moins d'un an $(39$ p. 100); des résultats similaires ont été obtenus dans la province de l'Adamaoua après la campagne de 1991 (38 \pm 3 p. 100), et celle du Nord après la campagne de $1992(46 \pm$ p. 100) $(6,7)$. Ce taux s'accroît considérablement avec l'âge des animaux.

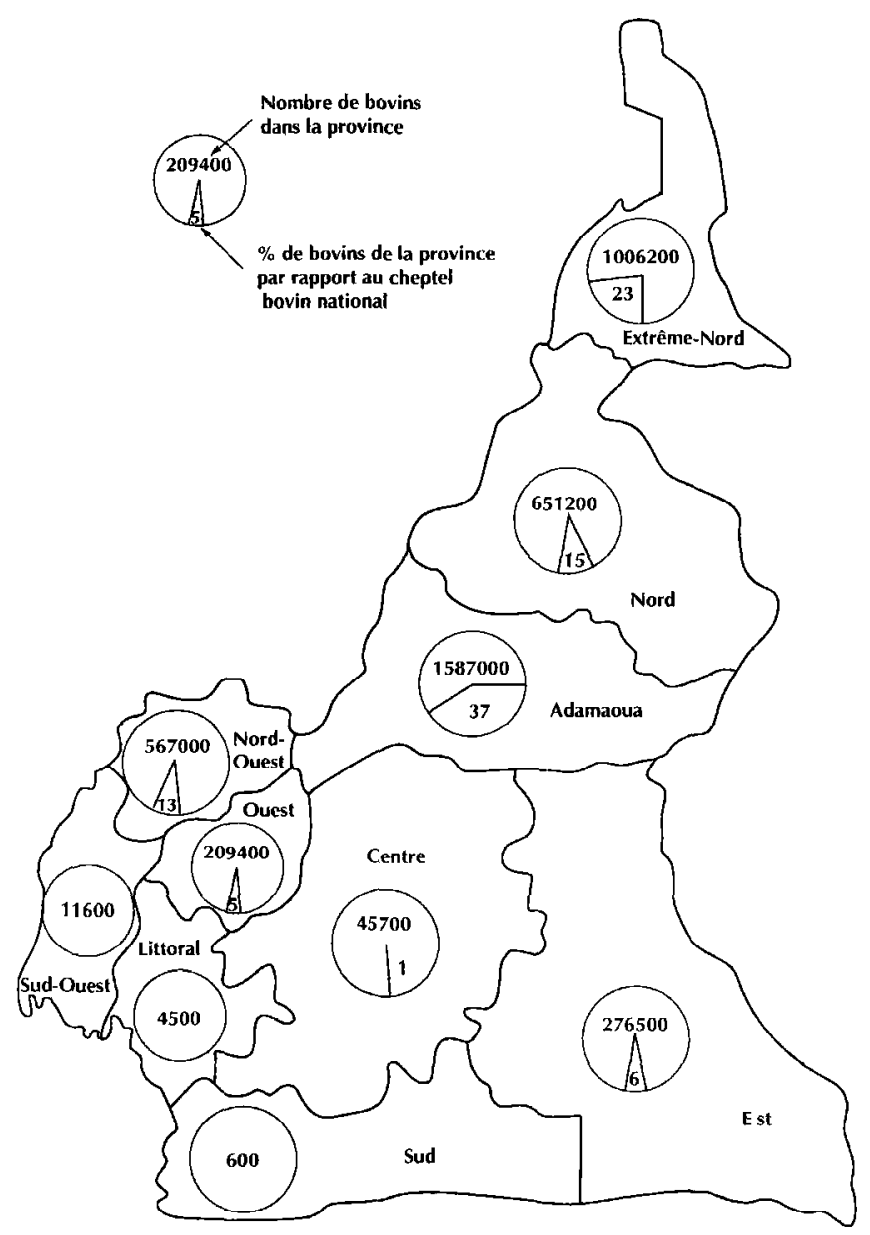

Figure 1 : répartition du cheptel dans les provinces.

Les différences de prévalence en anticorps anti-peste bovine sont significatives entre les tranches d'âge de 1 à 2 ans et de 2 à 3 ans ( chi-2 $=149,2 ;$ d.d.1 $=1 ; \mathrm{p}<0,05)$.

\section{Dans les troupeaux}

Le tableau III montre la prévalence en anticorps anti-peste bovine dans les différents troupeaux sondés. Quarante cinq troupeaux (16 p. 100) sont protégés (taux d'immunité glohale supérieure à 90 p. 100) (9).

Les différences de protection entre les troupeaux des six provinces sont statistiquement significatives $($ chi- $2=21,2$; $($ d.d.1. $=5$; $p<0,05)$.

Evaluation des anticorps contre le virus de la peste des petits ruminants (PPR) dans la population bovine à faible taux d'anticorps bovipestiques

Le tableau IV présente la répartition des animaux porteurs d'anticorps anti-virus de la PPR par province et par tranche d'âge ; le tableau $\mathrm{V}$ montre la prévalence globale en anticorps anti-PPR dans les différentes provinces.

Le pourcentage de bovins ayant séroconverti vis-à-vis de la PPR est faible parmi les animaux négatifs en anticorps bovipestiques. 
TABLEAU I

Répartition de l'immunité par province et par tranche d'âge

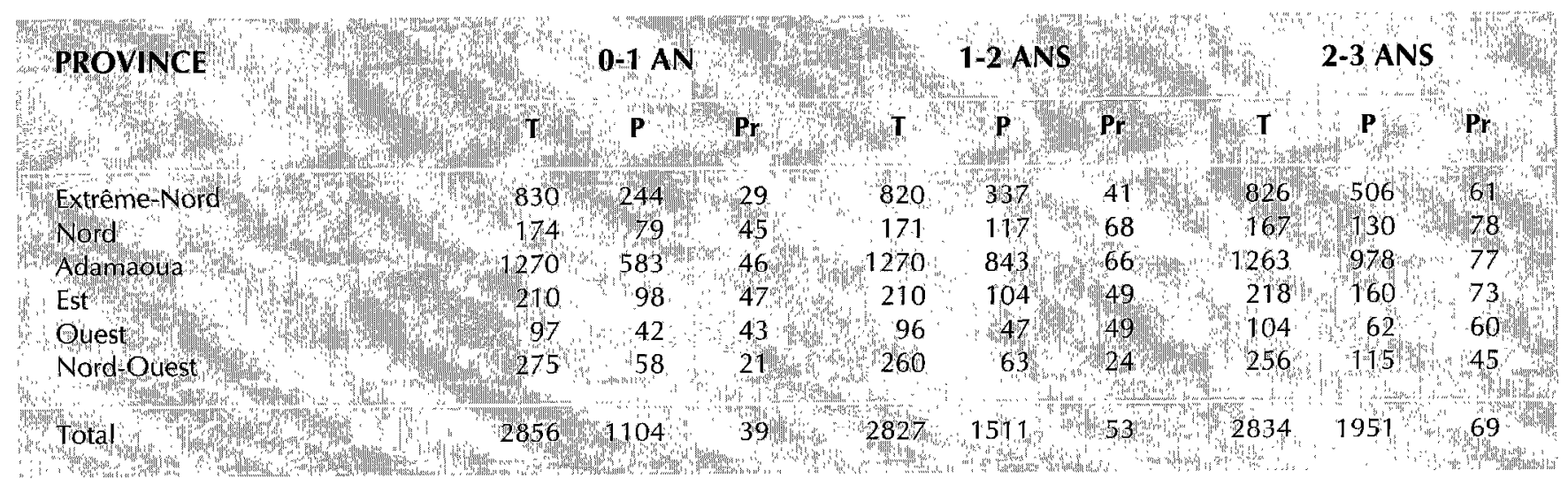

$\mathrm{T}=$ Testés $; \mathrm{P}=$ Positifs $; \mathrm{Pr}=$ Prévalence

TABIFAUI II

Immunité globale vis-à-vis de la peste bovine dans chaque province

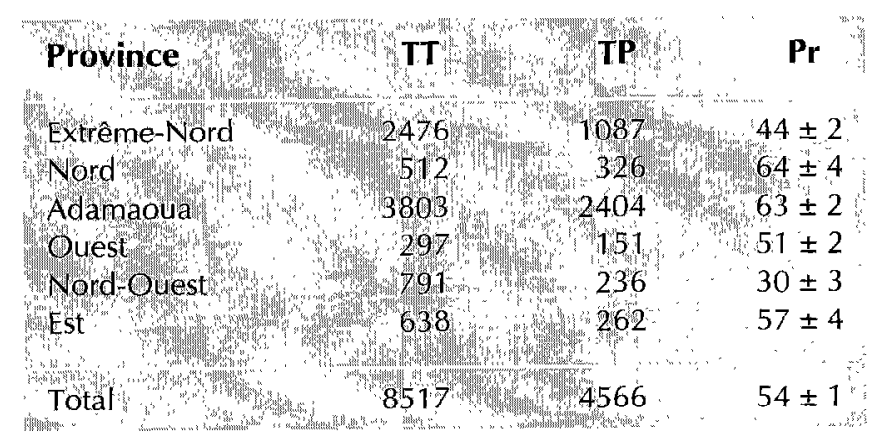

TT $=$ Total testés $; \mathrm{TP}=$ Total des sérums positifs $; \mathrm{Pr}=$ Prévalence
TABLEAU III

Répartition des troupeaux selon leur niveau de protection vis-à-vis de la peste bovine dans les provinces

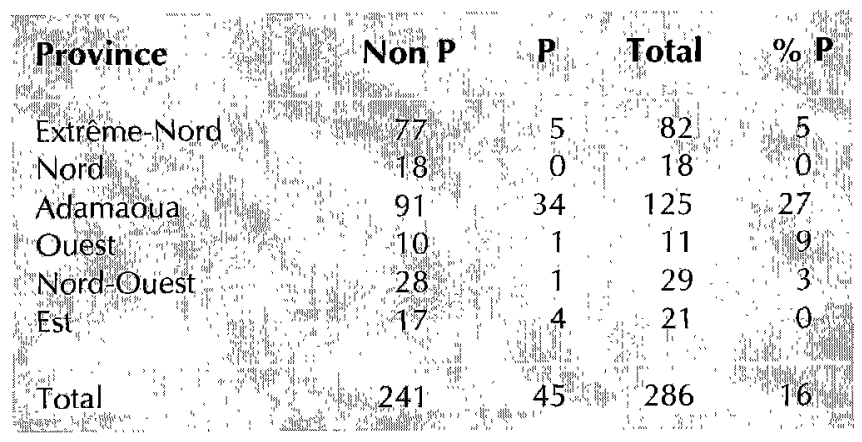

Non $\mathrm{P}=$ 'non protégés $; \mathrm{P}=$ protégés

TABLEAU IV

Répartition de l'immunilé contre la PPR par province et par tranche d'âge chez les bovins seronégatifs vis-à-vis de la peste bovine

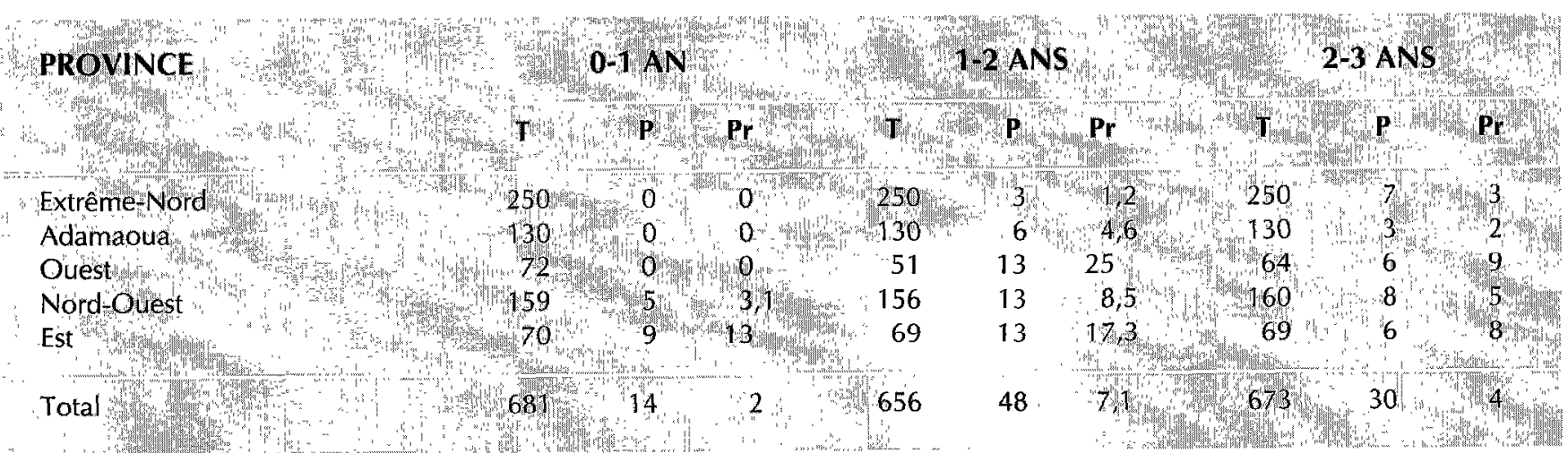

$\mathrm{T}=$ Testés $; \mathrm{P}=$ positifs $; \mathrm{Pr}=$ Prévalence 
TABLEAU $V$

\section{Prévalence de la PPR chez les bovins séronégatifs vis-à-vis de la peste bovine dans les différentes provinces}

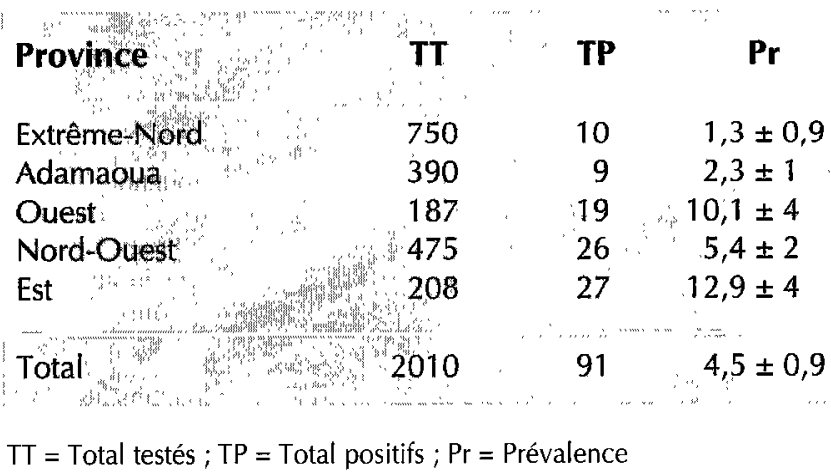

\section{DISCUSSION}

L'enquête sérologique a porté sur les classes d'âge dites "sensibles" vis-à-vis de la peste bovine. Les animaux âgés de plus de trois ans, généralement immunisés contre cette affection, ont été exclus du sondage.

Quatre provinces (Centre, Sud, Sud-Ouest, Littoral) n'ont pas été retenues pour l'enquête en raison de leur faible effectif en cheptel bovin.

Les deux provinces (Adamaoua, Nord) ayant fait l'objet de deux enquêtes successives n'ont pas enregistré de meilleurs résultats malgré les recommandations émises pour améliorer l'efficacité des campagnes de vaccination $(6,7)$.

Comme les années précédentes, les jeunes bovins sont moins protégés. Plusieurs raisons peuvent être avancées pour expliquer cette situation :

- la chance pour un animal d'avoir été vacciné augmente avec l'âge ;

- certains veaux, nés entre septembre et janvier, sont trop jeunes pour être vaccinés ou n'étaient pas encore nés lors du passage des équipes de vaccination ; ils échappent ainsi à la vaccination et s'avèrent négatifs en anticorps bovipestiques à l'examen sérologique ;

Un troupeau est considéré comme protégé si 90 p. 100 au moins des animaux qui le composent possèdent des anticorps bovipestiques. Par ailleurs, un cheptel est considéré comme protégé si 90 p. 100 au moins des troupeaux qui le constituent sont protégés (9). Au regard de ces données, aucune des six provinces visitées n'ćtait protégée.

Les provinces du Nord, de 1'Adamaoua enregistrent les meilleurs résultats bruts (64 et 63 p. 100 respectivement), tandis que les résultats les moins bons sont enregistrés dans les provinces de l'Extrême-Nord, de l'Est, du Nord-Ouest, de l'Ouest (44, 57, 30, 51 p. 100 respectivement).

Ces résultats sont inquiétants, particulièrement en ce qui concerne les provinces du Nord-Ouest et de l'Extrême-Nord : ce sont des régions à haut risque d'explosion de foyers d'origine exogène en raison de leur position frontalière, de l'absence de contrôle des mouvements d'animaux de part et d'autres des frontières et de la faiblesse du taux d'immunité post-vaccinale dans les pays voisins (Niger, Nigeria, Tchad, République centrafricaine, avec 46, 48, 58, 60 p. 100 d'immunité respectivement) $(2,10)$.
Le dynamisme plus ou moins grand des services vétérinaires présents sur le terrain, ajouté aux multiples raisons préalablement évoquées $(6,7)$ peuvent contribuer à expliquer le faible pourcentage d'animaux protégés contre la peste bovine observé dans certaines provinces.

La prévalence en anticorps anti-PPR est faible chez les bovins ne présentant pas d'anticorps bovipestiques et n'augmente pas de manière significative le niveau de protection du cheptel vis-à-vis de la peste bovine. D'autre part, l'infection des bovins par le virus de la PPR ne peut être évoquée pour expliquer la faiblesse de la couverture immunitaire post-vaccinale conire la peste bovine.

$\mathrm{Au}$ vu de ces résultats, quelques recommandations s'imposent :

- il semble nécessaire de re-dynamiser les campagnes de vaccination en portant une attention particulière aux jeunes bovins qui constituent une classe sensible ;

- la fourniture de moyens logistiques quand ils font défaut, la surveillance et la discipline dans l'utilisation des moyens de conservation sous froid ainsi que l'utilisation de la solution molaire de sulfate de magnésium comme solvant du vaccin devraient permettre une amélioration quantitative et qualitative de la vaccination contre la peste bovine (8) ;

- des campagnes d'information par l'intermédiaire de la presse ou de la radio nationale et locale favoriseraient la sensibilisation des éleveurs et pourraient les convaincre de faire vacciner tous leurs troupeaux ;

- les responsables directs des campagnes d'information devraient aussi pouvoir s'appuyer sur les autorités administratives (gouverneurs, préfets, sous-préfets, chefs de district) s'ils rencontraient des difficultés dans l'exécution de leur tâche ;

- ces responsables devraient également rendre compte par écrit du déroulement des campagnes de vaccination.

\section{CONCLUSION}

En 1993, dans le cadre du PARC, l'évaluation de l'immunité postvaccinale contre la peste bovine a été étendue à six provinces à grand élevage bovin.

Cinquante-quatre pour cent des animaux sont protégés contre cétle affection. Les provinces de L'Extrême-Nord, de l'Est, du NordOuest, de l'Ouest enregistrent les résultats les moins bons (44, 57, 30,51 p. 100 respectivement).

La prévalcnce en anticorps anti-PPR est faible dans les troupeaux bovins ne présentant pas d'anticorps bovipestiques. De même, la prévalence en anticorps anti-PPR n'augmente pas de manière significative le niveau de protection immunitaire contre la peste bovine. Le faible taux d'immunité post-vaccinale vis-à-vis de la peste bovine n'est certainement pas dû à l'infection des bovins par le virus de la peste des petits ruminants, mais probablement à une mauvaise exécution des campagnes de vaccination contre la peste bovine.

Des mesures doivent être prises pour améliorer les résultats.

\section{Remerciements}

Les auteurs remercient le Fonds européen de Développement (FED) qui a financé ce travail. Ils remercient également la Division AIEA/FAO, le CIRAD-EMVT pour la fourniture des kits ELISA. Ils expriment leur reconnaissance au Dr Lise Guerre pour son aide. Ils expriment également leur reconnaissance à tous les responsables des services d'élevage sur le terrain, pour leur aimable collaboration. 


\section{BIBLIOGRAPHIE *}

1. ANDERSON J., ROUX L.W, 1983. Use of an enzyme linked immunosorbant assay for the detection of IgG antibodies to rinderpest in epidemiological surveys. Res. vet. Sci., 34 (1) : 73-80.

2. BIOCH N., DIALLO I., 1990. Enquête sérologique dans un pays sahélien, le Niger. Problèmes d'échantillonnage et résultats de la sérosurveillance de la peste bovine. Revue Elev. Méd. vét. Pays trop., 43 (3) : 305-311.

3. LIBEAU G., PREHAUD C., LANCELOT R., COLAS F., GUERRE L., BISHOP D.H.L., DIALLO A., 1995. Development of a competitive ELISA for detecting antibodies to the peste des petits ruminants virus using a recombinant nucleoprotein. Res. vet. Sci, 58: 50-55.

4. Joint FAO/IAEA Division, 1991. Rinderpest ELISA Kit. Vienne, Autriche AIEA, 42 p.

5. MINISTERE DE L'ELEVAGE, DES PECHES ET DES INDUSTRIES ANIMALES, 1988. Rapport annuel des délégations provinciales 1986/87. Yaoundé, Cameroun, MINEPIA, $247 \mathrm{p}$.

6. NGANGNOU A., ZOYEM N., 1994. Evaluation de la protection vaccinale contre la peste bovine au Cameroun. I. La province de I'Adamaoua. Revue Elev. Méd. vét. Pays trop., 47 (1) : 35-37.

\section{Summary}

Ngangnou A., Zoyem N., Hamet M., Abdoulkadiri S. Evaluation of vaccinal protection against rinderpest in Cameroon. III. General evaluation

The national veterinary Laboratory, Garoua (Cameroon) has been carrying oul rinderpest sero-surveillance since 1989 as part of an effort made by the Panafrican Rinderpest Campaign (PARC) to control rinderpest in Africa. In 1993, 8517 serum samples collected from 286 cattle herds (from 0 to 3 years old) randomly chosen from six provinces with large cattle population (Far-North, North, Adamaoua, East, West, NorthWest) were tested using the rinderpest competitive ELISA technique: the herd immunity level was $54 \%$. Oul of 2010 serum samples from 68 non-protected cattle herds tested using the peste des petits ruminants (PPR) FIISA technique, 91 samples were positive $(4.5 \%)$ : this does not significantly increase the cattle immunity level against rinderpest. Significant differences in the immunity rates between provinces were observed. Suggestions to increase the immunity level are discussed.

Key words: Cattle - Rinderpest - Peste des petits ruminants Survey - Vaccine - ELISA - Cameroon.
* Cette bibliographie regroupe les références des parties I et II de cet article parues dans la Revue d'Elevage et de Médecine vétérinaire des Pays tropicaux (respectivement $47: 35-37$ et $48: 236-238$ ).
7. NGANGNOU A., ZOYEM N., 1995. Evaluation de la protection vaccinale contre la peste bovine au Cameroun. II. La province du Nord. Revue Elev. Méd. vét. Pays trop., 48 (3) : 236-238.

8. PROVOST A., BORREDON C., 1974. Un vaccin mixte antibovi-pestique-antipéripneumonique lyophilisé utilisable, sur le terrain, sans réfrigération. II. Utilisation du vaccin sur le terrain. Revue. Elev. Méd. vét. Pays trop., 27 (3) : 251-263.

9. TYLER L., 1989. Estimation de la prévalence d'anticorps du virus de la peste bovine. Nairobi, Kenya, OUA/IBAR, p. 10-45.

10. AlEA, 1994. The sero-monitoring of rinderpest throughout Africa, phase II. Results for 1993. In: proceedings of the FAO/IAEA/SIDA/OAU/IBAR/PARC coordinated Research Programme organized by the FAO/IAEA Division of nuclear Techniques in Food and Agriculture, Cairo, Egypt, 7-11 November 1993. Vienne, Austria, AIEA, $275 \mathrm{p}$.

11. AIEA, 1989. Section de la production et de la santé animales, division mixte OUA/BAR, campagne panafricaine contre la peste bovine. Principes directeurs pour la surveillance sérologique des bovins dans le cadre de la campagne panafricaine de lutte contre la peste bovine. Vienne, Austria, AIEA, $28 \mathrm{p}$.

12. SCHWARTZ D., 1969. Méthodes statistiques à I'usage des médecins et des biologistes. Paris, France, Flammarion, 306 p.

Reçu le 14.3.96, acuepté le 28.5.96

\section{Resumen}

Ngangnou A., Zoyem N., Hamel M., Abdoulkadiri S. Evaluación de la protección activa contra la peste bovina en Camerún. IIl Evaluación global

LANAVET, el laboratorio veterinario nacional en Garoua (Camerún) ha llevado a cabo una supervición serológica de rinderpest desde 1989, como parte de los esfuerzos realizados por el PARC (pan-african rinderpest), para controlar el rinderpest en el continente africano. En 1993, se recolectaron y examinaron 8517 muestras de suero, provenientes de 286 hatos escogidos por randomisación, en seis provincias con concentración elevada de ganado (Lejano-Norle, Norle, Adamaoua, Este, Oeste, Nor-Oeste). El nivel de inmunidad por hato alcanzó 54 p. 100. Se examinaron para anticuerpos del virus de la peste de los pequeños rumiantes, 2010 muestras de suero, provenientes de 68 hatos no protegidos. 91 muestras fueron positivas $(4,5$ p. 100). Esta cifra no basta para aumentar significativamente el nivel de protección del ganado contra rinderpest. El bajo nivel de immurnidad activa contra rinderpest no es debido a una infección por el virus de la PPR en el ganado. Existen diferencias significativas en las tasas de inmunidad entre las provincias. Se dan recomendaciones para aumentar el nivel de inmunidad.

Palabras clave : Ganado bovino - Peste bovina - Peste de los pequeños rumiantes - Encuesta - Vacuna - ELISA - Camerún. 